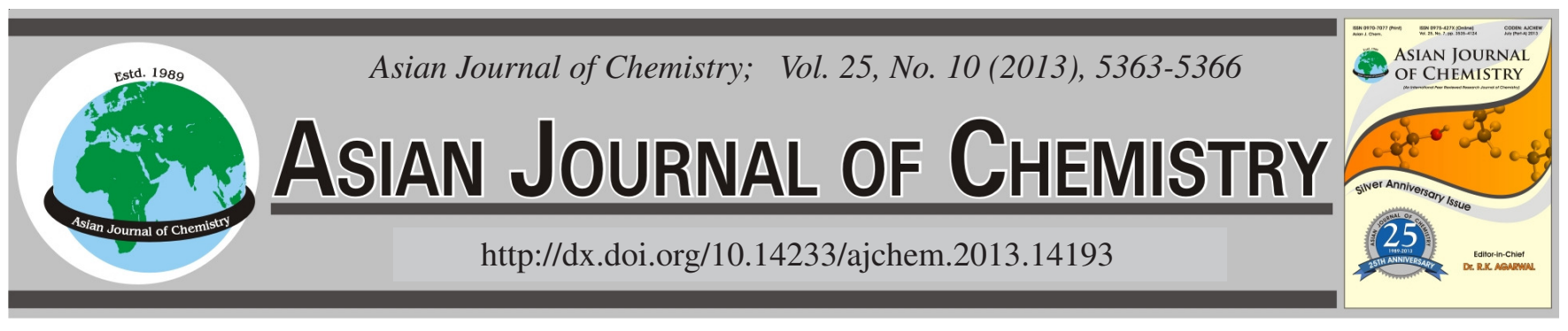

\title{
Chitosan-Gelatin-Pectin Composite Films from Polyion-Complex Hydrogels
}

\author{
X.F. ZHENG ${ }^{*}$ Q Q. LIAN and S.T. SONG
}

School of Physics and Chemistry, Hebei Normal University of Science and Technology, Qinhuangdao 066600, P.R. China

*Corresponding author: Tel: +86 24 2027029; E-mail: xuefang-zheng@163.com

(Received: 11 June 2012;

Accepted: 8 April 2013)

AJC-13206

Preparation and properties of composite films from gelatin, chitosan and pectin hydrogels are presented. Ionic interactions between
positively charged gelatin and negatively charged pectin produce reversible physical hydrogels with homogeneous molecular arrangement
that improve water resistance but not alter thermal stability relative to single polymer gels. The effect of preparation conditions to the
swelling rate and drug delivery of chitosan-gelatin-pectin films were also studied. Resulting interspersed permanent chemical hydrogel
showed a decreased swelling attitude with the increased dosage of crosslinker. However, further the films improved higher permeation in
simulated colonic fluid compared to the other media. The amount of bovine serum albumin released from the films at different time
intervals was estimated by UV spectrophotomertic method at $279 \mathrm{~nm}$. The dissolution profile and in vitro release kinetics showed that
chitosan-gelatin-pectin films were promising for controlled delivery of the drug. Formulation containing pectin, chitosan and gelatin may
be suitable as a coating formulation for colon targeted drug delivery.
Key Words: Pectin, Composite films, Swelling rate, Bovine serum albumin, Colonic delivery.

\section{INTRODUCTION}

The past few years have witnessd rapidly expanding interest in renewable feedstocks and marine food processing wastes as sources of biomolecules with potential to replace synthetic polymer ${ }^{1}$ in fabricating materials with biocompatibility, bioactivity, biodegradability for unique application ${ }^{2}$. Due to biomolecules have become more available, everincreasing demand for high-performance "natural" matrices for biomedical and pharmaceutical applications such as organ regeneration and tissue engineering ${ }^{3}$, wound dressing ${ }^{4}$, medical suture line, artifical limb, controlled drug delivery systems ${ }^{5,6}$, films, contact lenses and capsules for oral ingestion ${ }^{7}$, has stimulated design of smart matrices able to sense external changes ( $\mathrm{pH}$, temperature, ionic strength) and trigger release of active and bioactive compounds.

Pectin is a non-toxic water soluble gel-forming polysaccharide containing carboxylic acid groups. It is extracted from different sources, such as apple, pomelo, orange peel and so on. Pectin which exists in plant cell wall, the main unit is galacturonorhamnan and methylation half of lactose hyaluronic acid, is a kind of natural water soluble gather anion polysaccharide and can carry signal molecules and transmit many biological materials and also can serve as a kind of new biomedical materials. The properties of gelling have been identified as early as hundreds of years ago, but the production of commercial pectin only began in the early 20th century and the production of domestic pectin began in the 1980's.

Beacause of the $-\mathrm{COOH}$ containing in the pectin molecules and the $-\mathrm{NH}_{2}$ containing in the chitosan molecules, they will react each other to generate chitosan/pectin (CS/PT) polyelectrolytes compound hydrogels, whose structure appear in the corresponding response varying with environmental $\mathrm{pH}$, ionic strength and temperature, etc. Thus, they commonly can be used to the preparation of the film and the $\mathrm{pH}$ sensitivity microspheres and also can be used for food, health care products, drug loading and slow-release, etc. The three dimensional net shaped microporous structure gels will be composited with chitosan, gelatin and pectin, because of the functional group, which will have a lot of excellent performance.

This research selects chitosan, gelatin and pectin as the research object and the effect of crosslinking agent, $\mathrm{pH}$ value, ionic strength, temperature on swelling rate are studied and the effects of the performance of drug release are also investigated.

\section{EXPERIMENTAL}

Pectin, gelatin and chitosan (Deacelation degree $80 \%$ ) were purchased from Fuda chemical Co., in China; Glutaraldehyde (GA, $50 \mathrm{wt} \%$ in water) and glacial acetic acid were purchased from Across,Morris Plane, NJ; bovine serum albumin (BSA); buffer solution $(\mathrm{pH}=1.70,4.00,7.4,9.18)$. All commercially 
solvents and reagents were used without further purification. All other chemicals were of analytical grade.

Preparation of films: A series of films with 5.6 wt. \% total solid but different composition were generated mixing gelatin, pectin, and chitosan in different amount. Chitosan, gelatin and pectin (CS-Gel-PT) films were prepared in hot water $\left(60 \pm 0.5^{\circ} \mathrm{C}\right.$ containing $1 \mathrm{wt} \%$ acetic acid $)$ with vigorous stirring (1000 rpm) for $0.5 \mathrm{~h}$. After complete interdispersion of the solution, glutaraldehyde ( 2 wt \%) was added to the chitosan, gelatin, pectin solution, to get a series of different glutaraldehyde concentration solutions, under thorough and continuous mixing (550 rpm) one hour to crosslink the gelatin and form the hydrogels.

Once formed, all hudrogels were degassed and spread over the bottom of petri dishes (100 mm diameter) or polycarbonate rectangular templates (300 mm length $\times 150 \mathrm{~mm}$ width) depending on the specific successive analysis. Final films were obtained after evaporation of water in a vacuum oven at $40 \pm$ $0.5^{\circ} \mathrm{C}$ for $24 \mathrm{~h}$. Crosslinked films were additionally washed several times with Milli-Q water and then air-dried room temperature for $24 \mathrm{~h}$.

Scanning electron microscopy analysis (SEM): Strips of dry film (10 mm wide $\times 30 \mathrm{~mm}$ long) were immersed in $30 \mathrm{~mL}$ aliquots of $80 \mathrm{wt}$. \% ethanol water solution, with several changes. Then $80 \%$ ethanol was decanted and replaced with several changes of absolute ethanol. Next, strips were removed from absolute ethanol, quickly blotted dry and immersed in liquid nitrogen for $5 \mathrm{~min}$. The frozen strips were cross fractured manuslly using cold tweezers and the fractured pieces were thawed in absolute ethanol. Finally, the fragments were critical point dried from liquid $\mathrm{CO}_{2}$ and the dried fragments were glued to specimen stubs with Duco cement, sputter-coated with a thin layer of gold and examined with a Quanta 200 FEG scanning electron microscope, operated in the high vacuum, secondary electron imaging mode.

FT-IR analysis: FT-IR spectra were recorded using a Spectrum One spectrophotometer (NICOLET 200SXV FTIR, Perkin Elmer, USA) equipped with an Universal Attenuated Total Reflectance (UATR) device for tablet analysis in the spectral region $\left(4500-500 \mathrm{~cm}^{-1}\right)$.

Swelling behaviour: To evaluate the water sorption resistance of the CS-Gel-PT films, square piece of dry samples were weighed $\left(\mathrm{W}_{\mathrm{i}}\right)$ and then immersed in distilled water at $30{ }^{\circ} \mathrm{C}$ with shaking (100 rmp) for up to $24 \mathrm{~h}$. Swollen films were removed from water periodically, blotted dry and weighed $\left(\mathrm{W}_{\mathrm{f}}\right)$ to track sportion kinetics. The swelling rate (SR) was determined as described by others ${ }^{8}$.

$$
\text { Swelling rate }(\%)=\left[\frac{\left(\mathrm{W}_{\mathrm{f}}-\mathrm{W}_{\mathrm{i}}\right)}{\mathrm{W}_{\mathrm{i}}}\right] \times 100 \%
$$

Dissolution tests in vitro: Films samples $(0.5 \mathrm{~g} \pm 0.001)$ were accurately weighed and immersed in bovine serum albumin solution (BSA, $0.5 \mathrm{wt} \%$ ) at $0{ }^{\circ} \mathrm{C}$ for $25 \mathrm{~h}$. The swollen films loaded with drug were placed in a vacuum oven and dried under vacuum at $37^{\circ} \mathrm{C}$. The loading amount of drug in the films was calculated from the decrease in the concentration for the BSA solution $(279 \mathrm{~nm})$ which was determined using a UV spectrophotometer (UV-2401-PC, Shimadzu, Kyoto, Japan).
The kinetics of drug release were recorded using a Distek $^{\text {TM }}$ dissolution 2100 A paddle system (500 rpm, $37 \pm 0.5$ ${ }^{\circ} \mathrm{C}$ ) coupled with an UV Hewlett Packard spectrophotometer for detection of BSA and presented using the diffusion equation $^{9}$ as the ratio of the amount of drug released at the time $t$ $\left(\mathrm{M}_{\mathrm{t}}\right)$ /the total amount $\left(\mathrm{M}_{\text {inf }}\right)$ of the drug release from the films. The concenteation of BSA in the samples was calculated based on average calibration curves $(n=6)$. All dissolution studies were performed in triplicate ${ }^{10}$.

\section{RESULTS AND DISCUSSION}

Optimization of the films: According to previous research in this field, the variables affecting the ultimate swelling capacity of the films. After identification of the more effective parameters in the reaction, the optimum conditons for various experiments are shown in Table-1.

\begin{tabular}{ccccccc}
\multicolumn{7}{c}{ TABLE-1 } \\
\multicolumn{7}{c}{ REACTION COMPOSITION FOR THE } \\
PREPARATION OF OPTIMIZED SAMPLE \\
\hline Sample & $\begin{array}{c}\mathrm{m}_{\mathrm{CS}} \\
(\mathrm{g})\end{array}$ & $\mathrm{m}_{\mathrm{PT}}(\mathrm{g})$ & $\begin{array}{c}\mathrm{m}_{\mathrm{gel}} \\
(\mathrm{g})\end{array}$ & $\begin{array}{c}\mathrm{V}_{\mathrm{GA}} \\
(\mu \mathrm{L})\end{array}$ & $\begin{array}{c}\mathrm{V}_{\mathrm{CH}_{3} \mathrm{COOH}} \\
(\mu \mathrm{L})\end{array}$ & $\begin{array}{c}\mathrm{SR} \\
(\%)\end{array}$ \\
\hline $\mathrm{A}$ & 0.3 & 0.3 & 0.3 & 400 & 1000 & 743 \\
$\mathrm{~B}$ & 0.2 & 0.5 & 0.2 & 400 & 1000 & 463 \\
$\mathrm{C}$ & 0.5 & 0.2 & 0.2 & 400 & 1000 & 319 \\
$\mathrm{D}$ & 0.2 & 0.2 & 0.5 & 400 & 1000 & 418 \\
$\mathrm{E}$ & 0.40 & 0.1 & 0.40 & 400 & 1000 & 344 \\
\hline
\end{tabular}

After synthesizing the films following the conditions propose by the statistical method (five samples), the swelling rate of samples was evaluated. The ratio of $\mathrm{C}$ complexes formed membrane swelling degree is the smallest, that is in the pectin/chitosan/gelatine films the isoelectric point of gelatin, $-\mathrm{COO}^{-}$and $-\mathrm{NH}_{3}{ }^{+}$charge equal, mutual electrostatic attraction between strong, leading to swelling smaller. But the proportion of $\mathrm{C}$ is too thick, not easy to form a homogeneous composite membrane. Therefore, select the sample of D in the following composite membrane preparation process.

FT-IR analysis: FT-IR spectroscopy was used to ensure that the crosslinking reaction between the chitosan, pectin and gelatine had occurred. From the FT-IR spectral interpretation the following result were obtained. The FTIR of chitosan show intense band at $1595 \mathrm{~cm}^{-1}$ correspongding to the functional group $-\mathrm{NH}_{2}$ bending. The FT-IR of pectin show the band at $1732 \mathrm{~cm}^{-1}$ belongs to the $-\mathrm{COOH}$ stretching vibration peak. The absorption pesks at $1539 \mathrm{~cm}^{-1}$ can be assigned to the $-\mathrm{NH}_{2}$ bending vibration of gelatine. In contrast, after crosslinking, the vibrational band corresponding to primary amino groups at 1590 and $1539 \mathrm{~cm}^{-1}$ weaken and the vibrational band at $1732 \mathrm{~cm}^{-1}$ disappeared (Fig. 1), which prominent bands at $1428 \mathrm{~cm}^{-1}$ was ascribed to -COO- $\mathrm{NH}_{2}$. These results clearly confirmed that the crosslinking reaction had occured between the polymers.

SEM analysis: Composite membrane was revealed by scanning electron microscopy. SEM images of frozen-fractured surface in Fig. 2. Composite membrane with smooth surface and uniform has good biocompatibility in the composite membrane between chitosan, pectin and gelatin. 


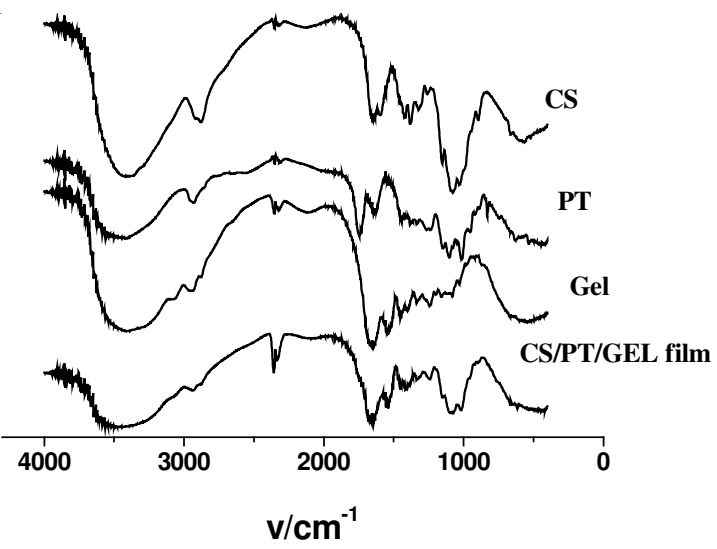

Fig. 1. FTIR of chitosan, pectin, gelatin and composition film

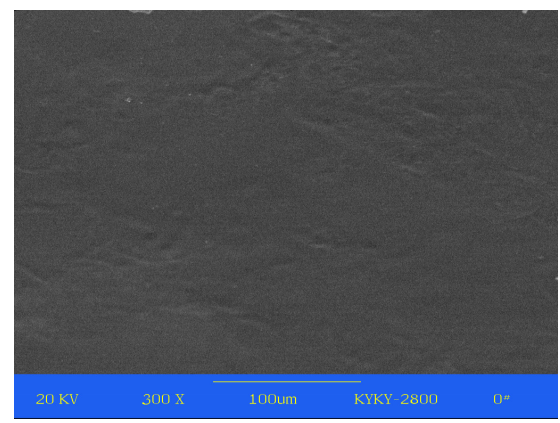

Fig. 2. SEM photograph of composite membrane

Effect of crosslinking agent on the swelling of composite membrane: Membrane swelling ratio reduced with increasing the amount of crosslinking agent in Fig. 3, this is because that the amount of crosslinking agent decreased, not only the molecular chain crosslinking density among chitosan/ gelatin/and pectin is low, but also the chain network structure of hydrogel molecular is loose and free space. Furthermore, there are strong hydrophilic interaction between $-\mathrm{OH},-\mathrm{COOH}$ and $-\mathrm{NH}_{2}$ groups, so the film interior can hold a lot of water and keep the larger degree of equilibrium swelling. However, when the amount of crosslinking agent increased, the crosslinking density of the film increases and the network structure and grid are reduced, therefore, water molecular inward permeability effective channel pore contract, so the equilibrium swelling degree reduced. But when the amount of crosslinking agent is more than $1 \mathrm{~mL}$, the film is more difficult to prepare, so the preparation with the amount of crosslinking agent is $1 \mathrm{~mL}$.

Effect of $\mathbf{p H}$ on membrane swelling properties: The $\mathrm{pH}$ is an important factor that can strongly influence on the swelling capacity of a film. At first, in order to survey the $\mathrm{pH}$ sensitivity of the film, swelling behaviour of the CS-Gel-PT film at $25^{\circ} \mathrm{C}$ in water with the $\mathrm{pH}$ ranging from 1.40-9.18 was investigated $^{11}$. The swelling rate of the film decreased in the $\mathrm{pH}$ rang between 1.40-7.40, but it it is increased with increasing $\mathrm{pH}$ from 7.4-9.18. The minimum swelling rate of the film was achieved at pH 7.4 (Fig. 4).

Swelling rate in different buffer solution with $\mathrm{pH}$ sensitivity is different, this is because of the polar groups such as $-\mathrm{COOH},-\mathrm{NH}_{2},-\mathrm{OH}$ and other else in the films and the difference of the degree of ionization. In the solution of $\mathrm{pH} 1.20$, $-\mathrm{NH}_{2}$ in CS-Gel-PT composite membrane is protonated into $-\mathrm{NH}_{3}{ }^{+}$, increased the electrostatic repulsion and hydrophilicity,

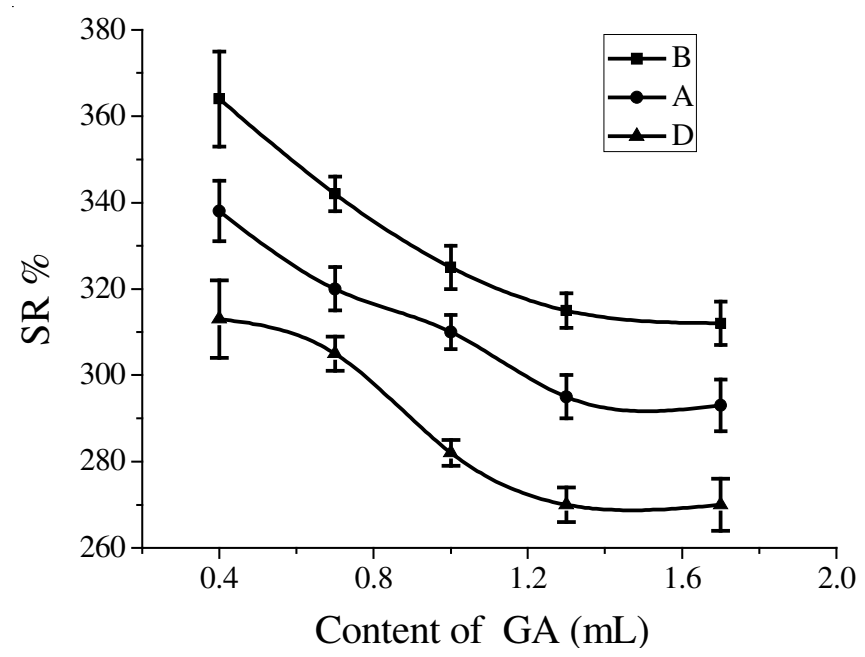

Fig. 3. Effect of crosslinking agent on the swelling of composite membrane

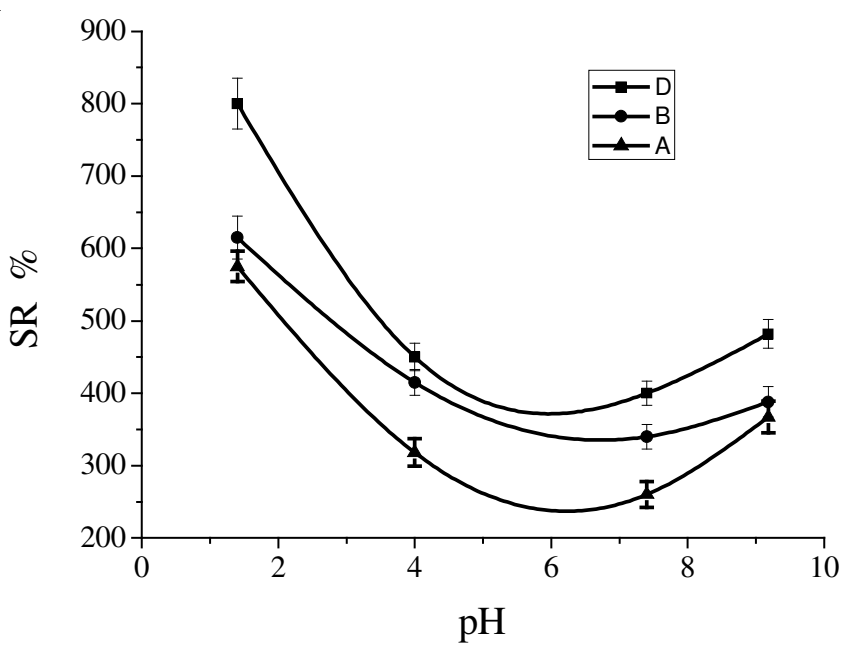

Fig. 4. Effect of $\mathrm{pH}$ on membrane swelling properties

leading to more swelling degree. At $\mathrm{pH} 4$, the swelling rate is lower, because that protonation degree and mutual electrostatic repulsion effect were weakened. In $\mathrm{pH} 7.4$ solution, composite membrane was in isoelectric point, thus swelling degree was minimum. In $\mathrm{pH}=9.18$, amino and hydroxyl hydrogen bonding effects lead to rise in swelling degree.

Effect of salt solution on the swelling rate: In comprise to the deionized water, the swelling of the absorbentd in saline solution was significantly decreased. This well-known phenomenon $^{12}$, commonly observed in the swelling of composite membrane, can be explained on the basis of osmotic pressure developed due to imbalanced distribution of ions in the polymer network and medium. The ionic attached to the polymer network are immobile and considered to be separated from the external solution by a semi-permeable membrane. While the film was placed in pure water, the maximum osmotic pressure is gained and thus the maximum water uptake is achieved. When the superabsorbent is in $\mathrm{NaCl}$ solution, the development of osmotic pressure is much lower due to presence of $\mathrm{Na}^{+}$and $\mathrm{Cl}^{-}$in the external solution. Therefore the swelling is rapidly declined. To investigate the influence of ionic strength on amount of water absorbency, the swelling in different $\mathrm{NaCl}$ solutions have been measured. The curve included in Fig. 5 showed that with increasing the ionic strength of the 
solution, the swelling capacity of composite membrane was significantly reduced ${ }^{13}$.

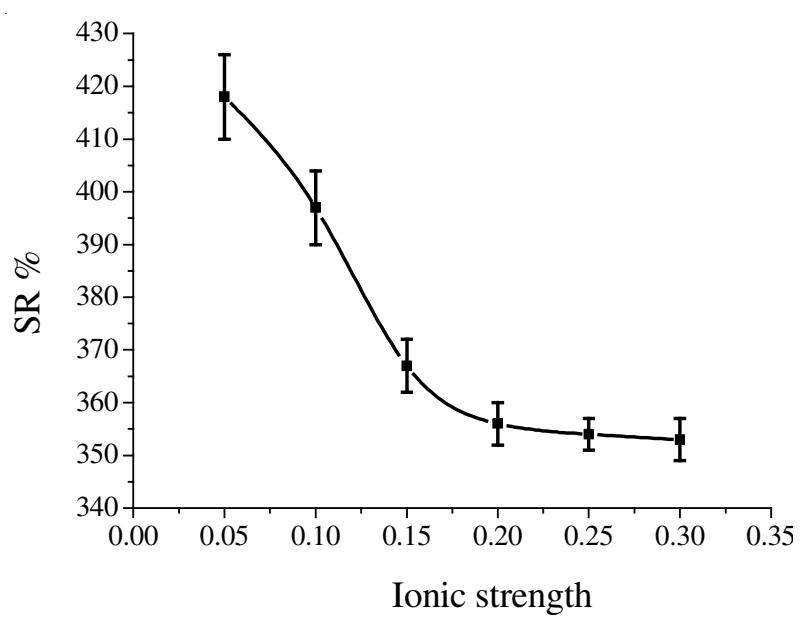

Fig. 5. Swelling rate of the CS-Gel-PT film in different $\mathrm{NaCl}$ solution

Drug release kietics from CS-Gel-PT composite membrane: The D as drug-loaded film carrier, was accurately weighed three part composite membrane of $\mathrm{D}$. The drug loading rate were $6.98 \times 10^{-3}, 6.82 \times 10^{-3}$ and $7.06 \times 10^{-3} \mathrm{~g} / \mathrm{g}$, after immersing in $0.5 \%$ (w \%) bovine serum albumin solution for $34 \mathrm{~h}$ in room temperature. The release kinetics of BSA from composite membrane in $\mathrm{pH} 1.2,4.0,7.4$ and 9.18 buffer solutions are presented in Fig. 6. The sample of D in $\mathrm{pH} 1.2$ solution rapidly disintegrated $\left(\mathrm{t}_{90} \%, 4 \mathrm{~h}\right)$, whereas those in other $\mathrm{pH}$ solution remained intact in aqueous medium. In $\mathrm{pH}$ 4-7.4 solutions, show relatively short release times $\left(t_{90} \%, 10-14 \mathrm{~h}\right)$. A longer release $(34 \mathrm{~h}$ ) was recorded for the $\mathrm{pH} 9.18$ buffer solution. This is attributed to a hydrophobic barrier limiting access of water and dissolution of the drug. According to peppas $^{14}$, there are three primary mechanisms by which the release of active agents can be controlled i.e., erosion, diffusion and swelling followed bu diffusion. Erosion may take palce via hydration by hydrolysis of the bulk, the polymer being slowly degraded starting at he periphery of the film. Diffusion can occur through the unhydrated polymer matrix but will generally be facilitated as the polymer gradually swells in contact with the body fluids. Therefore, composite membrane as a carrier for colonic drug delivery has been demonstrated and was useful to achieve the best $\mathrm{pH}$-dependent colonic drug delivery.

\section{Conclusion}

Chitosan-gelatin-pectin (CS-Gel-PT) composite films are of interest for use as excipients in controlled drug delivery systems. Hydrophobic interactions are belived to enhance the stability of interactions of chitosan, gelatin and pectin. It suggested that the release of drug is controlled by diffusion, or by swelling followed by diffusion, depending on both the degree of crosslinking.

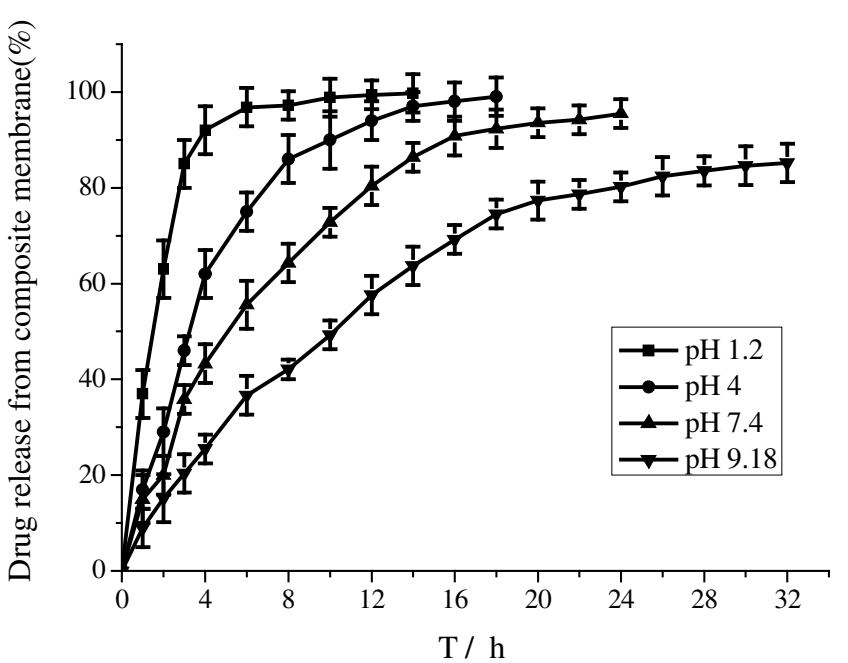

Fig. 6. Drug release kietics from CS-Gel-PT composite membrane in different $\mathrm{pH}$ buffer solution

\section{ACKNOWLEDGEMENTS}

The authors are grateful to Dr Shitao-Song for providing necessary facility to conduct the research work and also would like to thank Dongjun Wang for his valuable help during the course of the work. The authors acknowledged the financial support from Education Department of Hebei Province (No. 2012103000182).

\section{REFERENCES}

1. R.N. Tharanthan, Trends Food Sci. Technol., 14, 71 (2003).

2. C.C. Lin and A.T. Metters, Adv. Drug Delivery Rev., 58, 1379 (2006).

3. A. Khademhosseini and R. Langer, Biomater, 28, 5087 (2007).

4. S.Y. Ong, J. Wu, S.M. Moochhala, M.H. Tan and J. Lu, Biomater, 29, 4323 (2008).

5. A. Ghaffari, K. Navaee, M. Oshoui, K. Bayati and M. Rafiee-Tehrani, Eur. J. Pharm. Biopharm., 67, 175 (2007).

6. X. Wei, N. Sun, B. Wu, C. Yin and W. Wu, Int. J. Pharm., 318, 132 (2006).

7. A.S. Hoffiman, P.S. Stayton, O. Press, N. Murthy, C.A. Lackey and C. Cheung, Polym. Adv. Technol., 13, 992 (2002).

8. D. Myung, D. Waters, M. Wiseman, P.E. Duhamel, J. Noolandi and C.N. Ta, Polym. Adv. Technol., 19, 647 (2008).

9. N.A. Peppas, Pharm. Acta Helv., 60, 110 (1985).

10. E. Ojoe, E.M. Miyauchi, T.C. Viviani and V.O. Consiglieri, Braz. J. Pharm. Sci., 41, 377 (2005).

11. G.R. Bardajee, A. Pourjavadi and R. Soleyman, Nucl. Instrum. Methods Phs. Res. A, 266, 3932 (2008).

12. S.B. Teli, G.S. Gokavi and T.M. Aminabhavi, Sep. Purif. Technol., 56, 150 (2007).

13. A. Pourjavadi, H. Ghasemzadeh and R. Soleyman, J. Appl. Polym. Sci., 105, 813 (2007).

14. L.B. Peppas, Med. Plast. Biomater., 4, 34 (1997). 